\title{
Cardiovascular and Cerebrovascular Events After Parathyroidectomy in Patients on Renal Replacement Therapy
}

\author{
Kerstin M. Ivarsson ${ }^{1} \cdot$ Shahriar Akaberi $^{2} \cdot$ Elin Isaksson $^{3} \cdot$ Eva Reihnér $^{4} \cdot$ Tomasz Czuba $^{5}$ • \\ Karl-Göran Prütz $^{6} \cdot$ Naomi Clyne $^{2} \cdot$ Martin Almquist $^{7}$
}

\begin{abstract}
Background A majority of patients with end-stage renal disease suffer from secondary hyperparathyroidism, which is associated with osteoporosis and cardiovascular disease. Parathyroidectomy (PTX) is often necessary despite medical treatment. However, the effect of PTX on cardio- and cerebrovascular events (CVE) remains unclear. Data on the effect of PTX from population-based studies are scarce. Some studies have shown decreased incidence of CVE after PTX. The aim of this study was to evaluate the effect of PTX on risk of CVE in patients on renal replacement therapy.

Methods We performed a nested case-control study within the Swedish Renal Registry (SRR) by matching PTX patients on dialysis or with functioning renal allograft with up to five non-PTX controls for age, sex and underlying renal disease. To calculate time to CVE, i.e., myocardial infarct, stroke and transient ischemic attack, control patients were assigned the calendar date $(d)$ of the PTX of the case patient. Crude and adjusted proportional hazards regressions with random effect (frailty) were used to calculate hazard ratios for CVE.

Results The study cohort included 20,056 patients in the SRR between 1991 and 2009. Among these, 579 patients had undergone PTX, 423 during dialysis and 156 during time with functioning renal allograft. These patients were matched with 1234 dialysis and 736 transplanted non-PTX patients. The adjusted hazard ratio (HR) with $95 \%$ confidence interval (CI) of CVE after PTX was 1.24 (1.03-1.49) for dialysis patients compared with non-PTX patients. Corresponding results for patients with renal allograft at $d$ were HR (95\% CI) 0.53 (0.34-0.84).

Conclusions PTX patients on dialysis at $d$ had a higher risk of CVE than patients without PTX. Patients with renal allograft at $d$ on the other had a lower risk after PTX than patients without PTX.
\end{abstract}

Electronic supplementary material The online version of this article (https://doi.org/10.1007/s00268-019-05020-z) contains supplementary material, which is available to authorized users.

Kerstin M. Ivarsson

kerstin.ivarsson@med.lu.se

1 Clinical Sciences, Lund University, Alwallhuset, Barngatan 2A, 22185 Lund, Sweden

2 Department of Nephrology, Skane University Hospital Lund, Lund, Sweden

3 Department of Urology, Skane University Hospital Malmö, Malmö, Sweden
4 Department of Molecular Medicine and Surgery, Karolinska University Hospital, Stockholm, Sweden

5 National Registry Centre, Skane University Hospital Lund, Lund, Sweden

6 Department of Internal Medicine, Helsingborg Hospital, Helsingborg, Sweden

7 Department of Surgery, Skane University Hospital Lund, Lund, Sweden 


\section{Introduction}

Patients with end-stage renal disease (ESRD) often suffer from secondary hyperparathyroidism [1] (sHPT). SHPT is common both in patients on dialysis and in transplanted patients [2] and is associated with interstitial and vascular calcifications [3], cardiovascular disease, high-turnover bone disease [4] and mortality [5]. It can also contribute to calcification of allografts and thereby lead to deterioration of the transplant function [6]. In recent years, new medical treatments of sHPT have been introduced, with promising results $[7,8]$.

The rate of parathyroidectomies (PTX) has varied over time [9], but PTX is still necessary in patients with severe and/or therapy-resistant sHPT [10]. Reduction in cardiovascular calcification [11] and improvement in blood pressure [12], anemia and serum lipids [13] have been described after PTX. Further, PTX has been associated with an increase in bone density [14], and PTX also seems to improve survival in patients on dialysis [15-21]. Knowledge of the effect of PTX on risk of CVE is scant. Most [22-25] but not all [26] the previous studies reported lower risk of CVE after PTX. The previous studies have been performed on patients on dialysis, and the effect of PTX on risk of CVE among transplanted is still unknown. The aim of this study was to investigate the effect of PTX on the risk of CVE in patients on chronic renal replacement therapy, including both patients on dialysis and patients with renal allograft, in a nationwide population-based cohort.

\section{Methods}

\section{Study patients}

A matched case-control study was conducted on all patients in the Swedish Renal Registry (SRR) [27] between January 1, 1991, and December 31, 2009. Registration in the SRR is mandatory for all patients starting renal replacement therapy (RRT).

This study was performed and reported according to the Strengthening the Reporting of Observational Studies in Epidemiology (STROBE) statement [28].

\section{Definitions}

By linking SRR to the National Patient Register (NPR) with records of all procedure and diagnoses codes from hospital admissions [29], and using the operation code of total or subtotal parathyroidectomy at hospital discharge, we were able to define the occurrence and date of PTX. We also compared the dates for PTX in the NPR with information from the Scandinavian Quality Registry for Thyroid, Parathyroid and Adrenal Surgery (SQRTPA).

We defined CVE as myocardial infarction, stroke and transient ischemic attack (TIA), through discharge diagnoses registered in the NPR, Supplement Table 1. We also used discharge diagnoses to construct comorbidity index according to Charlson [30], by the algorithm described by Quan et al. [31]. The Charlson comorbidity index predicts the 1-year mortality for patients with one or more comorbid conditions, and the algorithm by Quan et al. translates these conditions into ICD codes. All incident PTX patients were identified in SQRTPA and/or in the NPR. The date of hospital admission in the NPR was used as date of PTX for all patients.

\section{Matching}

We divided the patients who had undergone PTX in two groups: those who were on maintenance dialysis treatment and those with a functioning renal allograft at the time of PTX. The definition of a patient with a functioning renal allograft is a transplanted patient who does not receive dialysis. The patients in each group were then randomly matched with one to five patients who did not undergo PTX during the study period. The matching criteria were birth year in 10-year categories (decades), sex and cause of ESRD in categories (autosomal dominant polycystic kidney disease, diabetes mellitus, glomerulonephritis, nephrosclerosis, pyelonephritis and other/unknown). Time to CVE was calculated by assigning both case and control patients the calendar date of PTX for the case patient, hereafter referred to as $d$. Patients not alive on $d$ were excluded from matching.

\section{Statistical analysis}

For continuous variables, means and standard deviations (SD) were calculated, while numbers and column percentages are presented for categorical variables. Patients were censored at death or at end of follow-up, which was December 31, 2009. Proportional hazards regression models with adjustment for random effect (frailty) [32] were used to compare hazard ratios (HR) between PTX and non-PTX patients in the matched sets. The frailty model handled the effect of multiple events. The timescale time from $d$ to censoring was used. Both crude and adjusted regressions were calculated.

Separate cox's regressions for PTX versus non-PTX patients, among those on dialysis or with functioning renal transplants, were performed. We adjusted for the following covariates: sex, time with functioning allograft (years), number of renal transplantations, cause of end-stage renal 
disease (autosomal dominant polycystic kidney disease, diabetes mellitus, glomerulonephritis, nephrosclerosis, pyelonephritis and other/unknown), Charlson comorbidity index score, time on RRT before $d$, time on dialysis before $d$ and CVE before $d$.

To test for non-proportional hazards or competing risks, a sensitivity analysis of the data with the pseudo value method [33] was made.

We considered results with $P<0.05$ statistically significant. Statistical analyses were made using STATA software version 12 (StataCorp LP, College Station, USA).

\section{Results}

\section{Patients and data}

There were 20,056 patients in the SRR between 1991 and 2009. Of these, we excluded 45 patients because of errors in reporting of patient information $(n=18)$ and censoring on the same day as initiation of RRT $(n=27)$. From the remaining 20,011 patients, 130 patients were excluded from matching because of PTX occurring before registration in the SRR.

In all, 590 patients had undergone PTX after registration in the SRR. Of these, 11 PTX patients could not be matched according to matching criteria and were excluded. Among the remaining 579 patients, 423 were on dialysis and 156 had a functioning renal allograft at the time of PTX. The patients in the two groups were matched with at least one and as many as possible up to five controls according to the criteria defined above. In total, the two groups of patients were matched with 1234 and 736 patients, respectively.

\section{Demographics and patient characteristics}

Demographics and patient characteristics of the cohort, PTX patients and matched non-PTX patients on dialysis and with functioning renal allograft are summarized in Tables 1, 2 and 3. Mean age of patients in the cohort population was higher than in the matched sets, both at initiation of RRT and at death. Cohort patients were more often male, more likely to have diabetes mellitus as cause of ESRD and had undergone fewer renal transplantations than the matched patients.

\section{Risk of cardio-/cerebrovascular events}

Among patients on dialysis at $d$, we found no significant difference in risk of CVE after PTX compared to control patients in the crude Cox regression, HR (95\% CI) 1.00 (0.84-1.19). After adjusting for sex, time with functioning
Table 1 Patient characteristics, whole cohort including the 2549 study patients

\begin{tabular}{lc}
\hline Factor & All patients $(N=20,011)$ \\
\hline Age (in years) at & \\
Start of RRT & $71.8(16.5)$ \\
Death $^{\mathrm{a}}$ & \\
Sex & $7131(35.6)$ \\
Female & $12,880(64.4)$ \\
Male & \\
Cause of ESRD & $1572(7.9)$ \\
ADPKD & $4843(24.2)$ \\
Diabetes mellitus & $3182(15.9)$ \\
Glomerulonephritis & $3651(18.2)$ \\
Nephrosclerosis & $1009(5.0)$ \\
Pyelonephritis & $5754(28.8)$ \\
Other and unknown & \\
Number of transplants & $14,951(74.7)$ \\
0 & $4686(23.5)$ \\
1 & $347(1.7)$ \\
2 & $23(0.1)$ \\
3 & $4(<0.1)$ \\
4 & $7045(35.2)$ \\
Alive at end of follow-up & \\
Follow-up time, months & $48.7(51.5)$ \\
\hline
\end{tabular}

Italicized numbers indicate mean (SD), numbers (percent)

$R R T$ renal replacement therapy, ESRD end-stage renal disease and $A D P K D$ autosomal dominant polycystic kidney disease

${ }^{\mathrm{a}}$ In 12,966 patients, death occurred before end of follow-up 31/12/ 2009

$\mathrm{b}_{31 / 12 / 2009}$

allograft, number of renal transplantations, cause of endstage renal disease, Charlson comorbidity index score, time on RRT before $d$, time on dialysis before $d$ and CVE before $d$, there was a higher risk of CVE after PTX, HR (95\% CI) 1.24 (1.03-1.49).

Shorter time with RRT before $d$ and polycystic kidney disease as underlying renal disease compared to other causes of renal disease were both associated with lower risk of CVD in patients on dialysis at $d$, (see Table 4).

In patients with renal allograft, there was a lower risk of CVE after PTX both in the crude model, HR (95\% CI) 0.62 (0.41-0.94) and in the adjusted model, HR (95\% CI) 0.53 (0.34-0.84) than for patients non-PTX patients. Short time specifically with renal transplant before/after $d$, a higher Charlson comorbidity score at $d$ and CVE before $d$ were associated with an increased risk of CVE (see Table 5).

In the test for non-proportional hazards or competing risks with pseudo values, the treatment effects were similar. As for the proportional hazards assumption, there was 
Table 2 Patient characteristics of the individually matched PTX and non-PTX patients, patients on dialysis at $d$

\begin{tabular}{|c|c|c|}
\hline & Parathyroidectomy (PTX) patients $(N=423)$ & Matched reference patients (no PTX) $(N=1234)$ \\
\hline \multicolumn{3}{|l|}{ Age (in years) at } \\
\hline Start of RRT & $51.6(14.7)$ & $53.8(14.4)$ \\
\hline $\operatorname{PTX}(d)^{\mathrm{a}}$ & $55.2(13.9)$ & $56.0(14.2)$ \\
\hline Death $^{\mathrm{b}}$ & $64.6(12.3)$ & $64.8(11.9)$ \\
\hline \multicolumn{3}{|l|}{ Sex } \\
\hline Female & $219(51.8)$ & $616(49.9)$ \\
\hline Male & $204(48.2)$ & $618(50.1)$ \\
\hline Time on RRT at PTX $(d)$ (years) & $3.6(3.2)$ & $2.2(2.4)$ \\
\hline \multicolumn{3}{|l|}{ Cause of ESRD } \\
\hline ADPKD & $60(14.2)$ & $173(14.0)$ \\
\hline Diabetes mellitus & $64(15.1)$ & $192(15.6)$ \\
\hline Glomerulonephritis & $122(28.8)$ & $363(29.4)$ \\
\hline Nephrosclerosis & $46(10.9)$ & $132(10.7)$ \\
\hline Pyelonephritis & $37(8.8)$ & $88(7.1)$ \\
\hline Other and unknown & $94(22.2)$ & $286(23.2)$ \\
\hline \multicolumn{3}{|l|}{ Number of transplants } \\
\hline 0 & $214(50.6)$ & $752(61.0)$ \\
\hline 1 & $167(39.5)$ & $422(34.2)$ \\
\hline 2 & $36(8.5)$ & $57(4.6)$ \\
\hline 3 & $5(1.2)$ & $3(0.2)$ \\
\hline 4 & $1(0.2)$ & \\
\hline Charlson comorbidity score at PTX $(d)$ & $1.4(1.7)$ & $1.6(1.8)$ \\
\hline Alive at end of follow-up ${ }^{c}$ & $233(55.1)$ & $604(48.9)$ \\
\hline Follow-up time, months from $d$ & $61.3(45.7)$ & $54.6(43.7)$ \\
\hline
\end{tabular}

Italicized numbers indicate mean (SD), numbers (percent)

$R R T$ renal replacement therapy, ESRD end-stage renal disease and ADPKD autosomal dominant polycystic kidney disease

${ }^{\mathrm{a}} d$, date of PTX or corresponding time for non-PTX patients

${ }^{\mathrm{b}}$ In 221/769 patients, death occurred before end of follow-up 31/12/2009

${ }^{c} 31 / 12 / 2009$

no statistically significant indication of this assumption not being fulfilled. Analyzing the data with a competing risk model also yields the same results.

\section{Discussion}

In this nationwide population-based study including 579 parathyroidectomized patients, we found a higher risk of CVE after PTX in patients on dialysis at the time of PTX, compared to patients without PTX. In contrast, the risk of CVE after PTX was lower in patients with a functioning renal allograft at the time of PTX compared to non-PTX patients.

There are five previous register studies investigating the risk of CVE after PTX compared to non-PTX patients, including only dialysis patients [22-26]. Four of them
[22-25] report a lower risk of CVE after PTX compared to non-PTX. In a population-based retrospective cohort study from Taiwan by Ma et al., the risk of acute coronary syndrome (ACS) after PTX was investigated. A total of 1047 PTX patients on dialysis with no previous renal transplantation or acute coronary syndrome, ACS, were matched with 4188 non-PTX patients. PTX patients had a significantly lower risk for ACS than non-PTX patients, with an adjusted HR (95\% CI) 0.74 (0.57-0.96) [22]. Further, in a cohort study by Lin et al., 53 non-diabetic patients on maintenance dialysis with severe SHPT (intact PTH $>800 \mathrm{pg} / \mathrm{ml}$ ) were followed for 72 months. One group of 23 patients had only medical treatment for SHPT due to unwillingness to receive the operation, while 30 patients also had PTX with autotransplantation. PTX was associated with both lower risk of major events (death, cerebrovascular events and myocardial infarcts) and better 
Table 3 Patient characteristics of the individually matched PTX and non-PTX patients, patients with functioning renal allograft at $d$

\begin{tabular}{|c|c|c|}
\hline & Parathyroidectomy (PTX) patients $(N=156)$ & Matched reference patients (no PTX) $(N=736)$ \\
\hline \multicolumn{3}{|l|}{ Age (in years) at } \\
\hline Start of RRT & $46.0(12.1)$ & $44.8(12.3)$ \\
\hline $\operatorname{PTX}(d)^{\mathrm{a}}$ & $51.1(12.3)$ & $50.5(12.1)$ \\
\hline Death $^{\mathrm{b}}$ & $62.3(14.3)$ & $59.7(10.4)$ \\
\hline \multicolumn{3}{|l|}{ Sex } \\
\hline Female & $78(50.0)$ & $359(48.8)$ \\
\hline Male & $78(50.0)$ & $377(51.2)$ \\
\hline Time on RRT at PTX $(d)$ (years) & $5.1(0.3)$ & $5.7(3.7)$ \\
\hline \multicolumn{3}{|l|}{ Cause of ESRD } \\
\hline ADPKD & $24(15.4)$ & $120(16.3)$ \\
\hline Diabetes mellitus & $25(16.0)$ & $125(17.0)$ \\
\hline Glomerulonephritis & $57(36.5)$ & $279(37.9)$ \\
\hline Nephrosclerosis & $11(7.1)$ & $43(5.8)$ \\
\hline Pyelonephritis & $11(7.1)$ & $30(4.1)$ \\
\hline Other and unknown & $28(17.9)$ & $139(18.9)$ \\
\hline \multicolumn{3}{|l|}{ Number of transplants } \\
\hline 1 & $131(84.0)$ & $674(91.6)$ \\
\hline 2 & $22(14.1)$ & $57(7.8)$ \\
\hline 3 & $3(1.9)$ & $4(0.5)$ \\
\hline 4 & & $1(0.1$ \\
\hline Charlson comorbidity score at PTX $(d)$ & $0.9(1.3)$ & $1.1(1.6)$ \\
\hline Alive at end of follow-up ${ }^{c}$ & $125(80.1)$ & $597(81.1)$ \\
\hline Follow-up time, months from $d$ & $76.9(51.1)$ & $73.5(49.9)$ \\
\hline
\end{tabular}

Italicized numbers indicate mean (SD), numbers (percent)

$R R T$ renal replacement therapy, ESRD end-stage renal disease and ADPKD autosomal dominant polycystic kidney disease

${ }^{a} d$, date of PTX or corresponding time for non-PTX patients

${ }^{b}$ In 221/769 patients, death occurred before end of follow-up 31/12/2009

c31/12/2009

laboratory status and blood pressure [23]. Costa-Hong et al. [24] came to the same conclusion in a study on 118 patients on maintenance hemodialysis unresponsive to medical treatment of SHPT on the waiting list for PTX. PTX was associated with both lower overall risk of death and occurrence of major cardiovascular events. Hsu et al. [25] finally found a lower risk of stroke in 1083 dialysis patients who underwent PTX compared with 1083 patients who did not, in their population-based cohort study, adjusted HR (95\% CI) 0.57 (0.41-0.79). Conzo et al. [26] on the other hand found no reduction in risk of cardiovascular morbidity, and the survival rate was unaffected by surgical treatment in their study of 30 PTX patients on dialysis compared with 20 non-PTX patients refusing the operation. Thus, our results for the dialysis group showing increased risk of CVE compared to non-operated patients are in contrast with most former studies. We also found a concluded lower risk of death for PTX patients on dialysis compared to the matched non-operated patients at $d$ [15].
In the present study, the risk of CVE after PTX was different in patients on dialysis compared to patients with a renal transplant at $d$. We observed an increased risk of CVE after PTX in patients on dialysis, which is in contrast to the studies reported above. In the comparing studies, there are some differences in study design and populations. Our study comprises all dialysis patients, not only those on hemodialysis as in the studies by Lin et al. [23], CostaHong et al. [24] and Conzo et al. [26].

There could be differences in overall health and social factors between patients on institutional dialysis and patients on peritoneal dialysis or home hemodialysis as in the prevalence of the different dialysis modalities in different populations. The lack of data on PTH levels in the study population makes the results difficult to compare to the results by Lin et al. [23] who included only patients with intact PTH $>800 \mathrm{pg} / \mathrm{ml}$. Moreover, our study period was longer than in any of the earlier studies, namely from 1991 to 2009, which comprises a period both before and 
Table 4 Relative risk of cardio-/cerebrovascular events for PTX patients compared with controls, on dialysis at $d^{\text {a }}(n=423 / 1234)$ [Cox proportional hazards regression and HR $(95 \% \mathrm{CI})]$

\begin{tabular}{lll}
\hline Factor & Unadjusted & Adjusted \\
\hline PTX & $1.00(0.84-1.19)$ & $1.24(1.03-1.49)$ \\
Sex men versus women & $1.13(0.84-1.53)$ & $1.21(0.92-1.58)$ \\
Time with functioning graft (year) & $0.30(0.24-0.39)$ & $0.53(0.39-0.62)$ \\
Number of transplantations & $0.38(0.31-0.45)$ & $0.50(0.40-0.62)$ \\
Cause of ESRD, ADPKD reference category & & $2.08(1.28-3.39)$ \\
Diabetes mellitus & $2.76(1.61-4.72)$ & $1.18(0.75-1.84)$ \\
Glomerulonephritis & $1.28(0.78-2.07)$ & $2.05(1.22-3.45)$ \\
Nephrosclerosis & $3.38(1.91-6.00)$ & $2.46(1.40-4.34)$ \\
Pyelonephritis & $2.14(1.15-3.98)$ & $1.39(0.87-2.21)$ \\
Other and unknown & $1.59(0.96-2.64)$ & $1.00(0.93-1.07))$ \\
Charlson score & $1.01(0.95-1.08)$ & $1.17(1.08-1.26)$ \\
Time on RRT before $d$ & $1.01(0.98-1.05)$ & $0.81(0.74-0.88)$ \\
Time on dialysis before $d$ & $1.01(0.96-1.05)$ & $2.28(1.90-2.75)$ \\
Cardio-/cerebrovascular events before $d$ & $2.80(2.33-3.35)$ & \\
\hline
\end{tabular}

The adjusted hazard ratios were adjusted for all the variables in the table

$R R T$ renal replacement therapy, ESRD end-stage renal disease and ADPKD autosomal dominant polycystic kidney disease

${ }^{\mathrm{a}}$ The date of PTX or corresponding date for non-PTX patients

Table 5 Relative risk of cardio-/cerebrovascular events for PTX patients compared with controls, with a functioning graft at $d^{\text {a }}(n=156 / 736)$ [Cox proportional hazards regression and HR $(95 \% \mathrm{CI})$ ]

\begin{tabular}{llr}
\hline Factor & Unadjusted & Adjusted \\
\hline PTX & $0.62(0.41-0.94)$ & $0.53(0.34-0.84)$ \\
Sex men versus women & $1.42(0.92-2.52)$ & $1.42(0.89-2.26)$ \\
Time with functioning graft (year) & $0.24(0.17-0.34)$ & $0.23(0.16-0.33)$ \\
Number of transplantations & $0.88(0.60-1.31)$ & $0.88(0.57-1.36)$ \\
Cause of ESRD, ADPKD reference category & & $1.62(0.74-3.52)$ \\
Diabetes mellitus & $1.80(0.75-4.32)$ & $0.95(0.47-1.92)$ \\
Glomerulonephritis & $1.12(0.51-2.44)$ & $1.46(0.51-4.17)$ \\
Nephrosclerosis & $1.81(0.59-5.62)$ & $0.82(0.26-2.61)$ \\
Pyelonephritis & $0.76(0.22-2.63)$ & $1.00(0.45-2.23)$ \\
Other and unknown & $1.15(0.48-2.78)$ & $1.14(1.02-1.27)$ \\
Charlson score & $1.61(1.05-1.28)$ & $0.96(0.90-1.02)$ \\
Time on RRT before $d$ & $0.98(0.93-1.04)$ & $0.99(0.86-1.13)$ \\
Time on dialysis before $d$ & $0.96(0.87-1.08)$ & $3.25(2.32-4.56)$ \\
Cardio-/cerebrovascular events before $d$ & $3.49(2.52-4.83)$ & \\
\hline
\end{tabular}

The adjusted hazard ratios were adjusted for all the variables in the table

$R R T$ renal replacement therapy, ESRD end-stage renal disease and ADPKD autosomal dominant polycystic kidney disease

${ }^{\mathrm{a}}$ The date of PTX or corresponding date for non-PTX patients

after the introduction of calcimimetics. By matching patients for calendar date of PTX, we ensured that both case and control patients in each matched set had the same medical option of treatment with cinacalcet. Thus, the option of cinacalcet treatment depended only on whether the calendar date was before or after the introduction of cinacalcet in Sweden.

The contrasting results from our former study of survival after PTX which showed HR (95\% CI) 0.80 (0.65-0.99) for dialysis patients who underwent PTX 
compared to non-PTX dialysis patients [15], and the present study with increased risk of CVE might be explained by the fact that early and multiple events after PTX not necessarily lead to mortality. Patients without PTX could have been exposed to more severe events and therefore had a higher mortality than the patients in the PTX-group. Another contributing factor could be that the prolonged survival time among PTX patients, presented in our former study, might increase the risk of having CVE at some point after PTX.

In recent years, more attention has been given to the fact that not only high levels of PTH but also very low levels of PTH are associated with increased mortality [34] and CVE $[35,36]$. One of the common complications of PTX is low levels of PTH after the procedure, so this could partly explain the higher risk of CVE for PTX patients. One could also speculate that patients without a functioning renal allograft might have higher levels of PTH than transplanted patients and therefore are more likely to be assigned total PTX than subtotal PTX. Total PTX is associated with lower levels of PTH postoperatively compared to subtotal PTX.

Our study design comprises all patients on RRT, not only those on dialysis but also transplanted patients, none of the other studies included that patient group. In our former study mentioned above, we investigated the survival after PTX compared to non-PTX patients for patients with renal transplants at $d$. We found no difference in survival between the two groups [15]. On the other hand, the risk of CVE was lower among PTX patients in the present study, as for the dialysis patients in the four studies above. Since there is a known risk of impaired transplant function after PTX [37], the operation is reserved for patients with severe sHPT [38], which might influence the risk of CVE after $d$.

There is evidence of negative effects of sHPT on the cardiovascular system. Mineral metabolism disturbances in calcium, phosphate and vitamin D following sHPT are associated with soft tissue calcification particularly in arteries, cardiac valves and myocardium [39]. Other mechanisms related to sHPT are suggested to lead to cardiovascular calcifications such as decreased calcium-sensing receptor expression on cardiovascular structures and a direct role of PTH in vascular calcifications through activation of type-1 PTH/PTHrP receptors [3].

Our study was limited by the lack of certain information. Although we performed matching and adjustment for a number of confounding factors, residual confounding cannot be ruled out. Access to laboratory data of PTH, plasma calcium, phosphate and creatinine in the study patients would have been valuable but were not recorded in the Swedish Renal Registry before 2005 and even after this date reporting was sporadic. Certain demographic and social data would probably also contribute to the analysis model, as would information on renal function, BK and cytomegalovirus, development of new-onset diabetes and relapse of underlying kidney disease among patients with functioning renal allograft.

There are certain strengths in the present study. The national registries that supplied our data contain information on almost all patients on RRT in Sweden between 1991 and 2009. Thus, this is the first truly population-based European study performed on cardio- and cerebrovascular outcome after PTX, and our findings ought to have high external validity and low bias depending on regional differences. Also, the long-term follow-up time includes different eras of PTX incidence and medical treatments.

In conclusion, in the present study, PTX was associated with higher risk of cardio-/cerebrovascular events after PTX for patients on maintenance dialysis. This was in contrast to some former studies. However, the risk was lower for patients with a functioning renal allograft at the time of PTX. The results of the present study need to be confirmed in other, large, prospective trials, preferably including data both on laboratory values and medication use, before any firm recommendations on PTX in relation to cardiovascular risk can be made.

Open Access This article is distributed under the terms of the Creative Commons Attribution 4.0 International License (http://crea tivecommons.org/licenses/by/4.0/), which permits unrestricted use, distribution, and reproduction in any medium, provided you give appropriate credit to the original author(s) and the source, provide a link to the Creative Commons license, and indicate if changes were made.

\section{References}

1. Llach F, Velasquez Forero F (2001) Secondary hyperparathyroidism in chronic renal failure: pathogenic and clinical aspects. Am J Kidney Dis 38(5 Suppl 5):S20-S33

2. Isaksson E, Sterner G (2012) Early development of secondary hyperparathyroidism following renal transplantation. Nephron Clin Pract 121(1-2):c68-c72

3. Torres PA, De Broe M (2012) Calcium-sensing receptor, calcimimetics, and cardiovascular calcifications in chronic kidney disease. Kidney Int 82(1):19-25

4. Cunningham J, Sprague SM, Cannata-Andia J, Coco M, CohenSolal M, Fitzpatrick L et al (2004) Osteoporosis in chronic kidney disease. Am J Kidney Dis 43(3):566-571

5. Ganesh SK, Stack AG, Levin NW, Hulbert-Shearon T, Port FK (2001) Association of elevated serum $\mathrm{PO}(4), \mathrm{Ca} \times \mathrm{PO}(4)$ product, and parathyroid hormone with cardiac mortality risk in chronic hemodialysis patients. J Am Soc Nephrol 12(10):2131-2138

6. Gwinner W, Suppa S, Mengel M, Hoy L, Kreipe HH, Haller H et al (2005) Early calcification of renal allografts detected by protocol biopsies: causes and clinical implications. Am J Transplant 5(8):1934-1941 
7. Verheyen N, Pilz S, Eller K, Kienreich K, Fahrleitner-Pammer A, Pieske B et al (2013) Cinacalcet hydrochloride for the treatment of hyperparathyroidism. Expert Opin Pharmacother 14(6):793-806

8. Suki WN, Zabaneh R, Cangiano JL, Reed J, Fischer D, Garrett L et al (2007) Effects of sevelamer and calcium-based phosphate binders on mortality in hemodialysis patients. Kidney Int 72(9):1130-1137

9. Akaberi S, Clyne N, Sterner G, Rippe B, Reihner E, Wagner P et al (2014) Temporal trends and risk factors for parathyroidectomy in the Swedish dialysis and transplant population-a nationwide, population-based study 1991-2009. BMC Nephrol 15:75

10. National Kidney Foundation K-DOQI (2007) Clinical practice guidelines for bone metabolism and disease in chronic kidney disease. Clin Rev Bone Miner Metab 5(1):53-67

11. Bleyer AJ, Burkart J, Piazza M, Russell G, Rohr M, Carr JJ (2005) Changes in cardiovascular calcification after parathyroidectomy in patients with ESRD. Am J Kidney Dis 46(3):464-469

12. Goldsmith DJ, Covic AA, Venning MC, Ackrill P (1996) Blood pressure reduction after parathyroidectomy for secondary hyperparathyroidism: further evidence implicating calcium homeostasis in blood pressure regulation. Am J Kidney Dis 27(6):819-825

13. Evenepoel P, Claes K, Kuypers D, Maes B, Vanrenterghem Y (2005) Impact of parathyroidectomy on renal graft function, blood pressure and serum lipids in kidney transplant recipients: a single centre study. Nephrol Dial Transplant 20(8):1714-1720

14. Stein MS, Packham DK, Wark JD, Becker GJ (1999) Response to parathyroidectomy at the axial and appendicular skeleton in renal patients. Clin Nephrol 52(3):172-178

15. Ivarsson KM, Akaberi S, Isaksson E, Reihner E, Rylance R, Prutz KG et al (2015) The effect of parathyroidectomy on patient survival in secondary hyperparathyroidism. Nephrol Dial Transplant 30(12):2027-2033

16. Kestenbaum B, Andress DL, Schwartz SM, Gillen DL, Seliger SL, Jadav PR et al (2004) Survival following parathyroidectomy among United States dialysis patients. Kidney Int 66(5):2010-2016

17. Foley RN, Li S, Liu J, Gilbertson DT, Chen SC, Collins AJ (2005) The fall and rise of parathyroidectomy in U.S. hemodialysis patients, 1992-2002. J Am Soc Nephrol 16(1):210-218

18. Iwamoto N, Sato N, Nishida M, Hashimoto T, Kobayashi H, Yamasaki S et al (2012) Total parathyroidectomy improves survival of hemodialysis patients with secondary hyperparathyroidism. J Nephrol 25(5):755-763

19. Goldenstein PT, Elias RM, Pires de Freitas do Carmo L, Coelho FO, Magalhaes LP, Antunes GL et al (2013) Parathyroidectomy improves survival in patients with severe hyperparathyroidism: a comparative study. PLoS ONE 8(8):e68870

20. Komaba H, Taniguchi M, Wada A, Iseki K, Tsubakihara Y, Fukagawa M (2015) Parathyroidectomy and survival among Japanese hemodialysis patients with secondary hyperparathyroidism. Kidney Int 88:350-359

21. Trombetti A, Stoermann C, Robert JH, Herrmann FR, Pennisi P, Martin PY et al (2007) Survival after parathyroidectomy in patients with end-stage renal disease and severe hyperparathyroidism. World J Surg 31(5):1014-1021. https://doi.org/10.1007/ s00268-006-0693-1

22. Ma TL, Chang RY, Chen HJ, Liu CY, Hsu CC, Hsu YH (2016) Risk of acute coronary syndrome after parathyroidectomy in patients with end-stage renal disease: a population-based cohort study in Taiwan. Nephrology (Carlton) 23:139-147
23. Lin HC, Chen CL, Lin HS, Chou KJ, Fang HC, Liu SI et al (2014) Parathyroidectomy improves cardiovascular outcome in nondiabetic dialysis patients with secondary hyperparathyroidism. Clin Endocrinol (Oxf) 80(4):508-515

24. Costa-Hong V, Jorgetti V, Gowdak LH, Moyses RM, Krieger EM, De Lima JJ (2007) Parathyroidectomy reduces cardiovascular events and mortality in renal hyperparathyroidism. Surgery 142(5):699-703

25. Hsu YH, Chen HJ, Shen SC, Tsai WC, Hsu CC, Kao CH (2015) Reduced stroke risk after parathyroidectomy in end-stage renal disease: a 13-year population-based cohort study. Medicine (Baltimore) 94(23):e936

26. Conzo G, Perna AF, Savica V, Palazzo A, Della Pietra C, Ingrosso D et al (2013) Impact of parathyroidectomy on cardiovascular outcomes and survival in chronic hemodialysis patients with secondary hyperparathyroidism. A retrospective study of 50 cases prior to the calcimimetics era. BMC Surg 13(Suppl 2):S4

27. Swedish Renal Registry. 11 March 2016. http://www.medscinet. net/snr/

28. STROBE statement. Strengthening the Reporting of Observational Studies in Epidemiology. 11 March 2016. http://www. strobe-statement.org/

29. The National Patient Register, NPR. 10 Nov 2017. http://www. socialstyrelsen.se/register/halsodataregister/patientregistret/ inenglish

30. Charlson ME, Pompei P, Ales KL, MacKenzie CR (1987) A new method of classifying prognostic comorbidity in longitudinal studies: development and validation. J Chronic Dis 40(5):373-383

31. Quan H, Sundararajan V, Halfon P, Fong A, Burnand B, Luthi JC et al (2005) Coding algorithms for defining comorbidities in ICD9-CM and ICD-10 administrative data. Med Care 43(11):1130-1139

32. Hougaard P (1995) Frailty models for survival data. Lifetime Data Anal 1(3):255-273

33. Parner ETAP (2010) Regression analysis of censored data using pseudo-observations. Stata J 10(3):408-422

34. Tentori FWM, Bieber BA, Karaboyas A, Li Y, Jacobson SH, Andreucci VE, Fukagawa M, Frimat L, Mendelssohn DC, Port FK, Pisoni RL, Robinson BM (2015) Recent changes in therapeutic approaches and association with outcomes among patients with secondary hyperparathyroidism on chronic hemodialysis: the DOPPS study. Clin J Am Soc Nephrol 10:98-109

35. Isaksson EAM, Seeberger A, Sterner G (2018) Is low pre-transplant parathyroid hormone a risk marker for cardiovascular disease in long-term follow-up of renal transplant recipients? Clin Exp Nephrol 22(5):1188-1197

36. Vadiveloo TDP, Leese CJ, Abraham KJ, Leese GP (2019) Increased mortality and morbidity in patients with chronic hypoparathyroidism: a population-based study. Clin Endocrinol 90(2):285-292

37. Schwarz A, Rustien G, Merkel S, Radermacher J, Haller H (2007) Decreased renal transplant function after parathyroidectomy. Nephrol Dial Transplant 22(2):584-591

38. Dewberry LC, Tata S, Graves S, Weber CJ, Sharma J (2014) Predictors of tertiary hyperparathyroidism: who will benefit from parathyroidectomy? Surgery 156(6):1631-1637

39. Shanahan CM, Crouthamel MH, Kapustin A, Giachelli CM (2011) Arterial calcification in chronic kidney disease: key roles for calcium and phosphate. Circ Res 109(6):697-711

Publisher's Note Springer Nature remains neutral with regard to jurisdictional claims in published maps and institutional affiliations. 